\title{
Optimization of post-classification processing of high-resolution satellite image: A case study
}

\author{
DONG Rencai, DONG Jiajia, WU Gang \& DENG Hongbing
}

\author{
State Key Lab of Systems Ecology, Research Center for Eco-Environmental Sciences, Chinese Academy \\ of Sciences, Beijing 100085, China \\ Correspondence should be addressed to Deng Hongbing (email: denghb@rcees.ac.cn) \\ Received October 10, 2005; accepted April 12, 2006
}

\begin{abstract}
The application of remote sensing monitoring techniques plays a crucial role in evaluating and governing the vast amount of ecological construction projects in China. However, extracting information of ecological engineering target through high-resolution satellite image is arduous due to the unique topography and complicated spatial pattern on the Loess Plateau of China. As a result, enhancing classification accuracy is a huge challenge to high-resolution image processing techniques. Image processing techniques have a definitive effect on image properties and the selection of different parameters may change the final classification accuracy during post-classification processing. The common method of eliminating noise and smoothing image is majority filtering. However, the filter function may modify the original classified image and the final accuracy. The aim of this study is to develop an efficient and accurate post-processing technique for acquiring information of soil and water conservation engineering, on the Loess Plateau of China, using SPOT image with $2.5 \mathrm{~m}$ resolution. We argue that it is vital to optimize satellite image filtering parameters for special areas and purposes, which focus on monitoring ecological construction projects. We want to know how image filtering influences final classified results and which filtering kernel is optimum. The study design used a series of window sizes to filter the original classified image, and then assess the accuracy of each output map and image quality. We measured the relationship between filtering window size and classification accuracy, and optimized the post-processing techniques of SPOT5 satellite images. We conclude that (1) smoothing with the majority filter is sensitive to the information accuracy of soil and water conservation engineering, and (2) for SPOT5 $2.5 \mathrm{~m}$ image, the $5 \times 5$ pixel majority filter is most suitable kernel for extracting information of ecological construction sites in the Loess Plateau of China.
\end{abstract}

Keywords: ecological construction, soil and water conservation measure, high spatial resolution satellite image, image post-processing, majority filter. 
In China, the Loess Plateau is a known area for severe soil and water loss ${ }^{[1-3]}$. In order to conserve water and soil and improve ecological environment, the Chinese government categorized the Loess Plateau as a key pilot area of constructing forest protection projects and has launched a huge afforestation movement since $1978^{[4,5]}$. In recent years, the Chinese government has launched a widespread ecological construction movement on the Loess Plateau, such as the Three Northern Regions (northeastern, northwestern and northern China), the Shelter Forest System Project, the Farmland Shelter Forest Projects, and the extensive program for transforming farmland into forests or grassland ${ }^{[6]}$.

Many soil and water conservation measures and engineering have been built on the Loess Plateau since 2001. In terms of the natural, economic, social conditions of these small basins, all soil and water conservation measures have been carried out in units appropriate to the area of small basins $\left(30 \mathrm{~km}^{2} \text { or less }\right)^{[3]}$. Because the primary property of loess terrain is its ability to maintain a vertical cliff, these manmade objects were ineluctably built in a functional relationship with the complicated surrounding. Generally, there are two main ways to control soil and water pouring into the Yellow River from the plateau area, one is planting vegetation, and the other is engineering measures ${ }^{[3]}$. The former is directly afforesting on the barren area. The latter needs building new artificial constructions, such as check-dam, contour vegetation barrier, rain water collectors, level step, etc. ${ }^{[7]}$, called soil and water conservation measures (SWCM). Monitoring ecological engineering is the basic need for appropriate and responsive management of these ecological construction projects. Hence, how to evaluate and monitor the vast amount of SWCM appears urgent for timely governance as it is physically difficult and labor intensive to investigate these objects in the field.

With the growing availability and accessibility of multi-spectral images, the application of remote sensing monitoring techniques has quickly become a valuable new method to detect land use changes. Furthermore, the recent emergence and application of high-spatial-resolution satellite images brings more reliable data resources for monitoring ecological engineering. Detailed geometric features can easily be recognized from high spatial resolution panchromatic images ${ }^{[8]}$.

It is anticipated that the development of the remote sensing monitoring techniques may contribute to the formalization of common methodological principles applicable to the conditions found on the Loess Plateau. The present research aims to determine appropriate methods for integrating imagery and geographical data within the target recognition process, thus enabling high-accuracy identification. This paper describes the development of image post-processing techniques and its application to SWCM on the Loess Plateau.

\section{Satellite image post-processing techniques}

Although remote sensing monitoring is suitable to supervise an ecological construction, acquisition of high spatial resolution images is only a partial solution. The images must then be translated into data products that are appropriate to, and that can be employed by, the relevant user communities (e.g., maps of land cover and land use for supervisors) ${ }^{[9]}$. 
SWCM are scattered and isolated pixel groups on the SPOT high-resolution image, so much as they look like noisy. In such a case, the use of high-resolution spatial data to extract information about SWCM requires adequate skills to reach a high accuracy. Thus, it is meaningful and also necessary to develop methods for fast acquisition of up-to-date SWCM information from satellite images.

Generally, some noisy data will appear as isolated pixels and small groups of pixels in classification remote sensing images. In other words, classified data often manifest in a salt-and-pepper appearance due to the inherent spectral variability encountered by a classifier when applied on a pixel-by-pixel basis. Therefore, it is sometimes desirable to aggregate local information in some way, e.g. in order to gain an overall picture of local cover type. The most commonly used approach involves the application of a moving window or $n$-by- $m$ pixel kernel, which is convolved with the image data ${ }^{[9]}$. The simplest and perhaps most common algorithm is majority filtering. Previous studies showed that smoothing image with majority filtering is effective for removing salt-and-pepper artifacts and eliminating noise in the post-classification of satellite images ${ }^{[10]}$.

Since the specific method for spatial aggregation of categorical data of this sort depends on the particular application and data input, we cannot use it blindly and optionally. As previous studies have also documented that spatial aggregation can lead to information loss or distortion of classified data ${ }^{[11]}$. For example, successive aggregations will typically lose scattered data of a certain class, but keep tightly clustered data ${ }^{[9]}$. Therefore, the influence of any resampling operation in a given data set depends not only on the specific method followed, but also on the quality of that data set. For this reason, we must test the correspondence between the structural properties and relations exhibited by the land cover parcels in the image.

To meet the primary goal of determining what kind of image post-processing techniques were more suitable for extracting accurate information on SWCM, an initial study was carried out with the main objective of developing a standard methodology to understand and explain the mechanism of error during image post-processing. The specific objectives of the study were to investigate the classification accuracies of different majority filtering and to select the parameter using knowledge-based rules and expert experience.

\section{Materials and methods}

\subsection{Study area}

The study area is in the area of the Zhaogou catchments, located at the eastern part of the Loess Plateau in Gongyi County, in the western part of Henan Province, China (Fig. 1). This area lies between $34^{\circ} 41^{\prime} 50^{\prime \prime}$ and $34^{\circ} 50^{\prime} 43^{\prime \prime}$ north latitude and $112^{\circ} 45^{\prime} 56^{\prime \prime}$ and $113^{\circ} 3^{\prime} 54^{\prime \prime}$ east longitude, $10 \mathrm{~km}$ east of Gongyi City and covering an area of $21.2 \mathrm{~km}^{2}$. There are significant topographic variations within the loess hills and gully landforms, with an average elevation of $80-280 \mathrm{~m}$ above sea level. The annual mean temperature and rainfall are $14.6^{\circ} \mathrm{C}$ and $583 \mathrm{~mm}$, respectively. The rainfall is concentrated mainly between July and September and varies greatly from year to year ${ }^{[12]}$. The soil in the study 
area is mainly derived from loess; it is fine in texture and weakly resistant to erosion. There are multiple species in this area such as Chinese date, P. guajava, D. quaesita, Paulownia, etc. The natural vegetation has been destroyed by cultivation, and due to ecological construction of recent years, many changes have taken place in the agricultural and forest landscape.

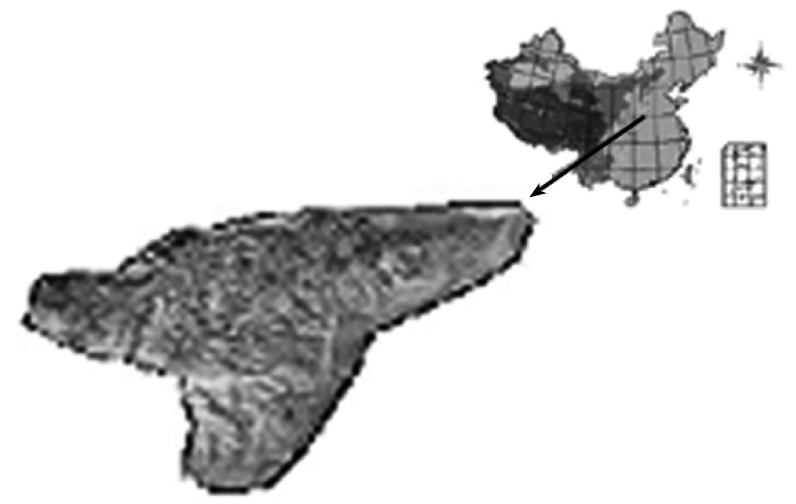

Fig. 1. Location and general situation of the study area on the Loess Plateau, China.

\subsection{Satellite image}

This project referenced two kinds of SPOT5 images (Scene ID: 5 276-280 02/11/08 03:06:42 $2 \mathrm{~T}$ ), which are multi-spectral image and panchromatic image. Both of them had been geometric and radiometric preprocessing by data dealer at Level 1A in WGS 84. The multi-spectral image consisted of four spectral bands (B1 to B4). B1 (green: 0.50$0.59 \mu \mathrm{m}$ ), B2 (red: $0.61-0.68 \mu \mathrm{m}$ ) and B3 (near infrared: $0.78-0.89 \mu \mathrm{m}$ ) had an initial resolution of $10 \mathrm{~m}$. Band $\mathrm{B} 4$ (mid-infrared, $1.58-1.75 \mu \mathrm{m}$ ) had a resolution of $20 \mathrm{~m}$ and was resampled at $10 \mathrm{~m}$ using a nearest neighbor algorithm. The panchromatic image had generated from SPOT5 Super-mode technique, which allows an image sampled at $2.5 \mathrm{~m}$ to be produced from two panchromatic images of $5 \mathrm{~m}$ resolution taken simultaneously by two High-Resolution Geometric (HRG) instruments ${ }^{[13]}$.

\subsection{Validation data}

In order to validate the final classification results, the checkpoint map was used to ground the truth data. The maps of checkpoints were generated in two ways: land-use maps and ground investigation data.

Land-use maps printed in 2000 by the local government have been digitized to acquire information of farmland, forest, grassland, water, etc. We selected some point located in this unchanged area as truth data. Using GPS, some monitoring points were collected and used to generate the validation map. We assessed the accuracy for all output maps.

\subsection{Methods}

Traditional image classifying techniques are divided into five key components ${ }^{[14,15]}$ : (1) Preprocessing of raw images; (2) classifying preprocessed images; (3) accuracy assessment; (4) post-classification processing; and (5) import classification result to GIS. 
This routine method ignores the change of accuracy of the final thematic image caused by image post-processing. In this study, the additional step to assess the accuracy of the post-classification image was inserted between (4) and (5).

(i) Pre-processing of raw images. With the ground control points identified from 1:10000 topological map and Digital Elevation Model (DEM), the panchromatic map has been corrected and the multi-spectral image was done. The total root mean square (RMS) error was limited in 0.4 pixels. The elevation information was required in the model for removing relief displacement. Panchromatic images with $2.5 \mathrm{~m}$ spatial resolution and multi-spectral images with 10 meters were fused.

(ii) Classifying preprocessed images. Land-cover classes were obtained for our monitoring purposes through referencing many thematic maps, such as vegetation maps, soil maps, investigation data of plots on the ground, and forest inventory databases. In the study area, there are forestland, farmland, shrub, grassland, and soil and water conservation measures (SWCM). Table 1 shows some characteristics of soil and water conservation measures identified on SPOT5. Because this study focuses on post-classification processing, we choose the common pre-classification, which is the supervised classification with a Maximum Likelihood Decision (MLD).

(iii) Accuracy assessment. Accuracy assessments were determined using standard error matrices to calculate overall accuracy ${ }^{[16,17]}$. Table 2 shows the classification error matrix.

Table 1 Identification criteria for soil and water conservation measures

\begin{tabular}{llll}
\hline \multicolumn{1}{c}{ Categories } & Tone & Texture & \multicolumn{1}{c}{ Pattern } \\
\hline Check dam & light & sharp & lie in the valley centre, down slope \\
Level step & light & smooth & linear feature \\
Rain water collectors & light & harsh & along the road, on the rooftop \\
Fish-scale pits & light grey & fine & two-dimensional fine pattern \\
\hline
\end{tabular}

Table 2 Classification error matrix of initial classification image

\begin{tabular}{lccccc}
\hline Class type & Forestland & Farmland & Grassland & Shrub & SWCM \\
\hline Forestland & 786 & 145 & 95 & 56 & 7 \\
Farmland & 136 & 872 & 69 & 76 & 16 \\
Grassland & 82 & 78 & 566 & 37 & 788 \\
Shrub & 36 & 66 & 18 & 36 & 25 \\
SWCM & 12 & 18 & 9 & 79.4 & 309 \\
$\%$ Corr. & 74.7 & 74.0 & 74.8 & & 83.1 \\
Overall accuracy & $=76.6 \%$, average class accuracy $=77.2 \%$, kappa coefficient $=0.73$ \\
\hline
\end{tabular}

(iv) Post-processing. As expected, numerous isolated pixel or noise pixels appear in classified images. Hence, post-classification smoothing with a majority filter was necessary to reduce unneeded detail and further improve the classification accuracy.

First, using the clump function processed the classified image. The clump function was used to label each pixel in the images with a value indicating the size of the contiguous cluster of pixels that contained the pixel. This function has 2 options for the way that pixel clusters can be defined: (1) only the adjacent 4 pixels in the cardinal directions are considered to be contiguous with the center pixel, or (2) all 8 neighbors of a given 
pixel are contiguous with the center pixel. It appeared, upon visual inspection of a subset image that was clustered by each method, that the definition of the neighborhood might significantly affect the resulting filtered image. Therefore, images were clumped with both 4 and 8 contiguous neighbors in this study.

Then, majority filtering was applied to the clumped images. In majority filtering, an $n$-by- $m$ window is centered about each pixel in a given image. The value that appears the maximum number of times among the values lying within the window is the determined value. This output is placed at the location of the center pixel, where the window was centered. This procedure is then repeated and applied to every pixel in the image. We filtered the initial classified image using the different windows size $(2 \times 2,3 \times 3,4 \times 4,5 \times 5$, $6 \times 6,7 \times 7,8 \times 8,16 \times 16,32 \times 32,64 \times 64,128 \times 128$ pixels by pixels), and attained individually improved classified maps, each of which was assessed individually.

(v ) Area changes analysis. From area histogram of each classified image, we calculated the area of each class type. Formula (1) was used to check the area difference comparing initial area of classified image.

$$
\Delta S i=\left(V_{i}-C_{i}\right) / C_{i},
$$

where $\Delta S$ is the area difference, $V_{i}$ is the area of post-filtered image, $C_{i}$ is the area of original image, and $i$ is the processing order of different window size.

(vi) Accuracy assessment of post-classification image. After post-processing by filtering with different window sizes, we assessed the post-classification images again. (The same approach is in step 3). All classification error matrixes were created.

\section{Results}

\subsection{Changes of the class type areas}

Fig. 2 shows the area change curve of all class types after majority filtering with different window sizes. Compared with the area of original classified image, the area of farmland and shrub class increases synchronously with the bigger filtering window size. The increase rate of farmland class's area is quicker than shrub class's. After $2 \times 2,4 \times 4$, $8 \times 8,128 \times 128$ pixels filter, the area of farmland class will rise by $0.17 \%, 0.58 \%, 1.26 \%$, $8.29 \%$, respectively. On the other hand, the area of forestland, grassland, and SWCM decrease with bigger filter window size. With particular emphasis on the and SWCM

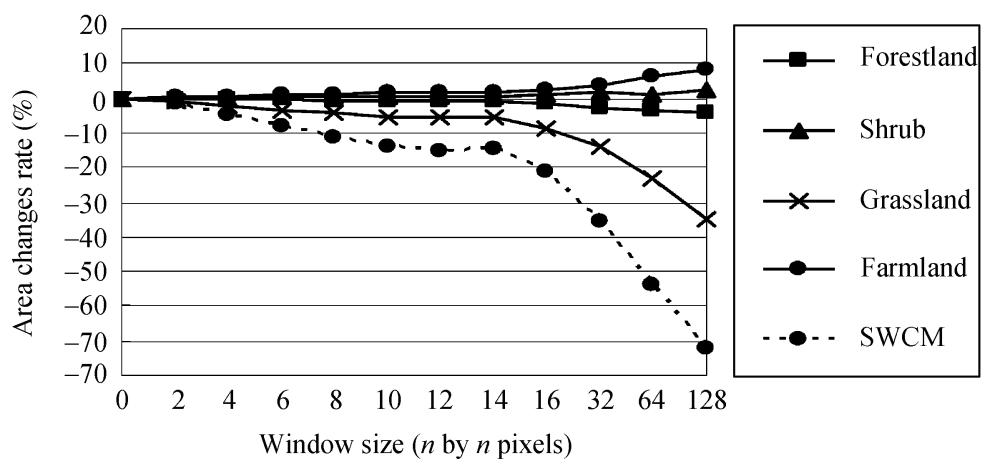

Fig. 2. Area changes of class type by the majority filtering with different window sizes. 
classes, after $128 \times 128$ pixel majority filtering, its area reduced to $27.85 \%$ of its original area.

For this study area, we observed that both farmland and shrub patch are consistent and dominant. On the contrary, the spatial patterns of forestland, grassland, and SWCM are fragmental and scattered. As a result, we think that the area of dominant class will increase and disadvantageous class will decrease with bigger filtering size.

\subsection{Image quality changes}

According to different window sizes, the original classified images were smoothed and the improved maps were generated, respectively. Fig. 3 shows some post-filtering images: (1) the original classified image, (2) majority filter by $5 \times 5$ pixels by pixels, (3) $16 \times 16$ pixels by pixels, and (4) $32 \times 32$ pixels by pixels. From visual inspection, we found that noisy pixels were reduced and more smoothed when the window size grew bigger. In the images from $5 \times 5$ pixels majority filter processing, we cannot find the isolated pixels and small groups of pixels. However, some known targets disappeared after the $16 \times 16$ majority filtering. In the final image of the $128 \times 128$ pixel majority filter, it is very difficult to find SWCM class; only forestland, grassland, shrub and farmland are left.

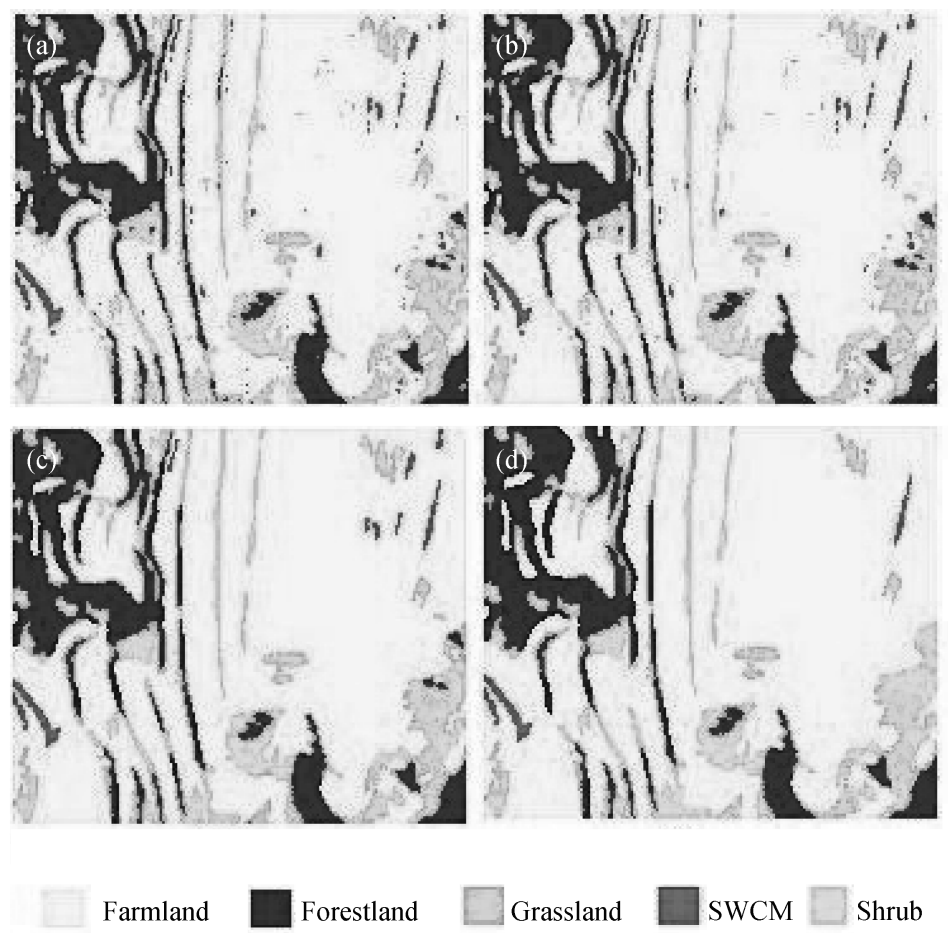

Fig. 3. Some images and distributions of class types after various window size majority filtering. (a) Original classified image (i.e., prior to filtering); (b) $5 \times 5$ pixels filtering; (c) $16 \times 16$ pixels filtering; (d) $32 \times 32$ pixels filtering.

\subsection{Accuracy variation}

In Fig. 4, with different window sizes majority filtering, the curves of overall and average accuracy variations were shown. We can see that both overall and average accuracy 
were influenced significantly by the post-processing technique. The result shows the same trends with the area change in section 3.1. Both the accuracy of forest types and grass type were decline gently and SWCM decreased dramatically form $88.6 \%$ to $8.9 \%$. In contrast, the accuracy of farmland and shrub increased from $72 \%$ to $99.6 \%$, and $74.4 \%$ to $92.2 \%$, respectively. For the curve of overall accuracy, the peak point existed while window size was between $4 \times 4$ and $6 \times 6$. Also the overall accuracy reached the peak point at a $5 \times 5$ pixel window size filtering.

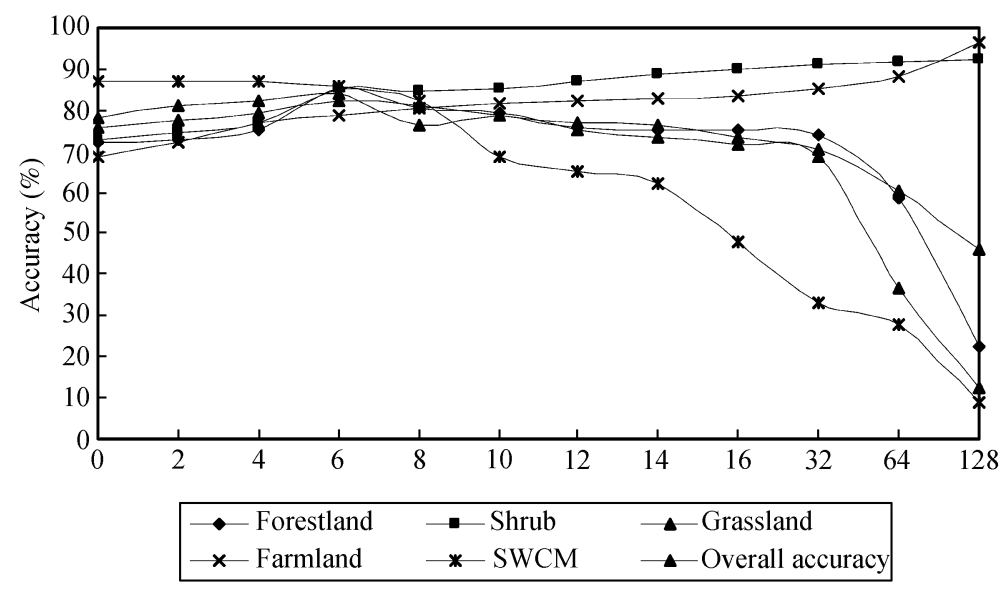

Fig. 4. Changes of overall and average class accuracy after majority filtering by various windows sizes.

\section{Discussion}

In this study, we find that different window sizes of image smoothing caused a difference in overall and average accuracy, i.e. image smoothing with majority filtering could generate misclassification. Compared with the original classified image, we find that: (1) the area change of SWCM class type decreased remarkably, while that of farmland and shrub classes increased; and (2) the final classification accuracy was influenced deeply by filter window size. This phenomenon is closely related to the traits and spatial pattern of the SWCM in this study area. Farmland and shrub pixel groups are continuous and aggregative in the satellite image with a $2.5 \mathrm{~m}$ spatial resolution, in clusters larger than $5 \times 5$ pixels. However, SWCM are discontinuous such that the classification detail of the parts of the image located in the valleys eroded at less then 6 pixels, i.e. 15 m. For example, rainwater collectors built under the cliff were a small pixel group surrounded by a weeded area. After smoothing with the majority filter at a $5 \times 5$ pixel window size, rainwater collectors in the farmland were removed, and farmland area was increased. Both level ditches and check-dams were also discontinuous pixel patterns. If the window's size were larger, the area of SWCM would be less and be replaced by farmland and shrub.

Smoothing with the majority filter is under 'Window Majority Rule', which determines the majority class value in a $5 \times 5$ pixels window and assigns it to the center pixel in the window. According to our investigation, most pixel groups of SWCM class type in this area are between $4 \times 4$ pixels $(10 \times 10 \mathrm{~m}$ size $)$ than $6 \times 6$ pixels $(15 \times 15 \mathrm{~m}$ size $)$. If the 
window size is larger than $6 \times 6$ pixels, most information about check-dams and rainwater collectors got filtered out. On the other hand, filtering at less than $4 \times 4$ pixels caused a lower overall accuracy, because most noisy pixels belong to isolated pixels, $2 \times 2$ pixels groups and $3 \times 3$ pixels groups. Therefore, it was crucial that the majority threshold was set to 25 pixels for distinguishing noisy pixels from target's pixels.

These effects are consistent with previous research in which dominant map classes increased and rare classes decreased, or disappeared entirely, when a simple majority filter was applied $^{[11,18]}$. For example, using the IRS WiFs (Wide Field Sensor) satellite data, the $3 \times 3$ majority filter was applied to removing salt-and-pepper appearance for the simulation of landscape pattern and ecological processes in southern California plant communities. The WiFs satellite data has two bands — Red and Near Infrared (NIR), with spatial resolution $188.3 \mathrm{~m}^{[10]}$. To develop an operational Landsat TM image classification protocol for Forest Inventory and Analysis (FIA) forest area estimation, the classified images were spatially post-processed using variations on a $3 \times 3$ majority filter and a clump and eliminate technique ${ }^{[19]}$. Using Landsat TM data $(30 \mathrm{~m})$ for vegetation map on the Loess Plateau, the $16 \times 16$ majority filter was suitable for removing salt-and-pepper pixels ${ }^{[20]}$. Furthermore, compared with the conventional window-based filtering method, an adaptive filtering method was developed. The noisy pixels are first located and then filtered based on the output of the pulse coupled neural network ${ }^{[21]}$.

For this study, depending on both visual inspection and accuracy assessment, we consider that the optimal window size is the peak point of overall accuracy. We determined the $5 \times 5$ windows size, where we can get the highest overall accuracy of $84.4 \%$ at those parameters, was the optimal parameter for image filtering. In other words, $5 \times 5$ pixel majority filtering is optimal threshold for the SPOT5 $2.5 \mathrm{~m}$ image soil and water conservation project on the Loess Plateau.

\section{Conclusions}

This case study has shown that extracting information on SWCM, using high spatial resolution satellite images to monitor the status of ecological construction, must pay more attention to image post-processing techniques. Different parameters resulted in different final classification accuracies. For different regions and diversity images, we need to optimize the image processing parameter by the knowledge-based methods. SWCM information will lessen with the majority filter. Tests against reference data indicated an optimal window size between 4 and 6 pixels. A $5 \times 5$ window was chosen for ease of application and to minimize bias.

Acknowledgements This research was supported by the National Natural Science Foundation of China (Grant No. 70325002) and the Knowledge Innovation Project of the Chinese Academy of Sciences (Grant No. KZCX3-SW-423).

\section{References}

1 Fu B J, Chen L D, Ma K M, et al. The relationships between land use and soil conditions in the hilly area of the loess plateau in northern Shaanxi, China. Catena, 2000, 39: 69-78 
2 Chen L D, Wang J, Fu B J, et al. Land-use change in a small catchments of northern Loess Plateau, China. Agric Ecos Environ, 2001, 86: 163-172

$3 \mathrm{Wu}$ B. Countermeasures and its evaluation on soil and water conservation in the Loess Plateau, China. Report on World Conference on Disaster reduction. 18-22 January 2005, Kobe, Hyogo, Japan. 2005

4 Qiao Y L, Wang Y, Tang J Y. Study of remote sensing monitoring of dynamic change of the Loess Plateau forest resources. Adv Space Res, 2004, 33: 302-306

5 Stolte J, Ritsema C J, Bouma J. Developing interactive land use scenarios on the Loess Plateau in China, presenting risk analyses and economic impacts. Agric Ecos Environ, 2004, 105: 387-399

6 Li W H. Degradation and restoration of forest ecosystems in China. For Ecol Manage, 2004, 201: $33-41$

$7 \mathrm{Xu} \mathrm{X} \mathrm{Z,} \mathrm{Zhong} \mathrm{H} \mathrm{W,} \mathrm{Zhang} \mathrm{O} \mathrm{Y.} \mathrm{Development} \mathrm{of} \mathrm{check-dam} \mathrm{systems} \mathrm{in} \mathrm{gullies} \mathrm{on} \mathrm{the} \mathrm{Loess} \mathrm{Plateau,} \mathrm{China.}$ Environ Sci Policy, 2004, 7: 79-86

8 Shi W Z, Zhu C Q, Tian Y, et al. Wavelet-based image fusion and quality assessment. Int J Appl Earth Obser Geo-infor, 2005, 6: 241-251

9 Barr S, Barnsley M. Reducing structural clutter in land cover classifications of high spatial resolution remotely-sensed images for urban land use mapping. Comput Geosci, 2000, 26: 433-449

10 Pradhan S. Regional land cover mapping of the Hindu Kush Himalayan (HKH) Region: an approach to understand the dynamics of land use and land cover change using satellite image. Available on line: http://www.gisdevelopment.net//, 2002

11 Syphard A D, Franklin J. Spatial aggregation effects on the simulation of landscape pattern and ecological processes in southern California plant communities. Ecol Model, 2004, 180: 21-40

12 Huang M B, Shao M G, Zhang L, et al. Water use efficiency and sustainability of different long-term crop rotation systems in the Loess Plateau of China. Soil Tillage Res, 2003, 72: 95-104

13 Pasqualini V, Martini C P, Pergent G, et al. Use of SPOT5 for mapping seagrasses: An application to Posidonia oceanica. Remote Sens Environ, 2005, 94: 39-45

14 ERDAS Inc. ERDAS Imagine Field Guide. Atlanta, GA. 1995

15 Prenzel B, Treitz P. Remote sensing change detection for a watershed in north Sulawesi, Indonesia. Prog Planning, 2004, 61: 349-363

16 Congalton R. A review of assessing the accuracy of classifications of remotely sensed data. Remote Sens Environ, 1991, 37: 35-46

17 Niel T G V, McVicar T R. Current and potential uses of optical remote sensing in rice-based irrigation systems: a review, Australian. J Agric Res, 2004, 55: 155-185

18 Moody A, Woodcock C E. The influence of scale and the spatial characteristics of landscapes on land-cover mapping using remote sensing. Landsc Ecol, 1995, 6: 363-379

19 Raptisa V S, Vaughana R A, Wrightb G G. The effect of scaling on landcover classification from satellite data. Comput Geosci, 2003, 29: 705-714

20 Tang G A, Zhang Y S, Liu Y M, et al. Remote Sensing Digital Image Processing (in Chinese). Beijing: Science Press, 2004. 232-233

21 Zhang J Y, Lu Z J, Shi L, et al. Filtering images contaminated with pep and salt type noise with pulse-coupled neural networks. Sci China Ser F-Inf Sci, 2005, 48(3): 322-334 